\title{
Upper bound estimators of the population size based on ordinal models for capture-recapture experiments
}

\author{
Marco Alfò $^{1}$ @ ～| Dankmar Böhning ${ }^{2}$ @ | Irene Rocchetti ${ }^{3}$
}

\author{
${ }^{1}$ Dipartimento di Scienze Statistiche, \\ Sapienza Università di Roma, Rome, Italy \\ ${ }^{2}$ Southampton Statistical Sciences Research \\ Institute, University of Southampton, \\ Southampton, UK \\ ${ }^{3}$ Consiglio Superiore della Magistratura, \\ Rome, Italy
}

Correspondence Marco Alfò, Sapienza Università di Roma, Rome, Italy.

Email:marco.alfo@uniroma1.it

\begin{abstract}
Capture-recapture studies have attracted a lot of attention over the past few decades, especially in applied disciplines where a direct estimate for the size of a population of interest is not available. Epidemiology, ecology, public health, and biodiversity are just a few examples. The estimation of the number of unseen units has been a challenge for theoretical statisticians, and considerable progress has been made in providing lower bound estimators for the population size. In fact, it is well known that consistent estimators for this cannot be provided in the very general case. Considering a case where capture-recapture studies are summarized by a frequency of frequencies distribution, we derive a simple upper bound of the population size based on the cumulative distribution function. We introduce two estimators of this bound, without any specific parametric assumption on the distribution of the observed frequency counts. The behavior of the proposed estimators is investigated using several benchmark datasets and a large-scale simulation experiment based on the scheme discussed by Pledger.
\end{abstract}

\section{K E Y W O R D S}

capture-recapture experiments, frequency of frequencies distribution, ordinal data, population size estimation, upper bound

\section{1 | INTRODUCTION}

Capture-recapture methods were originally developed in the ecological setting with the aim of estimating the unknown size of an (possibly elusive) animal population; since then, they have been gradually applied to other empirical settings, ranging from epidemiology and public health, see Böhning et al. (2004), to biodiversity, see Bunge et al. (2012), text analysis, see Efron and Thisted (1976), and software engineering, see Liu et al. (2015). An overview on closed population capture-recapture methods in given by Chao et al. (2001). For human populations, we usually observe individual records from multiple systems; that is, we have information in the form of an indicator variable $x_{i j}$ that is equal to 1 if the $i$ th unit has been recorded by the $j$ th system, $i=1, \ldots, N, j=1, \ldots, m$, and equal to 0 otherwise. The goal is to estimate the size $N$ of the population based on several, incomplete, lists of individuals recorded from that population; given the study design, the information is available only for those individuals with $x_{i}=\sum_{j=1}^{m} x_{i j}>0$, that is only for individuals that have been registered by at least one source. Being identified by a list (in human/social studies) corresponds to being captured on a sampling occasion (in wildlife studies); the probability of being recorded in a list will be referred to as the capture probability. Often, the number of available lists in human studies is lower than the number of trapping/sampling occasions in wildlife studies.

When the study output is summarized by a frequency of frequencies distribution, say $\left(x, n_{x}\right)$, where $n_{x}$ denotes the number of units that have been recorded exactly $x$ times, the problem of recovering the population size $N=\sum_{x \geq 0} n_{x}$ is equivalent to that of recovering the number of unrecorded units, $n_{0}$. By using a specific parametric model to fit the observed distribution, it is possible to derive an estimate for $n_{0}$ and, therefore, for $N$. However, it is well known, see Sanathanan (1977), that a consistent estimator for $n_{0}$ cannot be 
derived in the general family of nonparametric mixture densities. Choosing the best model has been proven to be a complex task, with no general solution, as several data-generating processes can provide the same fit to the observed, truncated, distribution, see Link (2003) for a thoughtful example. As a result, lower bound estimators have attracted particular interest, see Chao (1989) and Mao (2007); in fact, it is generally acknowledged that a finite upper bound for the population size does not exist, see Mao and Lindsay (2007).

By using a simple ordinal model to represent the observed frequency distribution, however, we show that the population size may be bounded from above, regardless of the true data-generating process. Unfortunately, the bound depends on unknown quantities referring to the complete (untruncated) distribution; therefore, we show how this can be approximated by using the observed frequency counts and give some guidance on conditions for such an approximation to work well.

The paper is structured as follows. In Section 3, we introduce the notation, the elementary treatment of the problem, and some basic relations that can be readily established; in Section 4 , an upper bound for $N$ is provided, which can be estimated by using the approximations given in Section 5. Section 6 discusses the application of the proposed estimators to five benchmark datasets. All of these datasets have the essential feature that the population size is known; we may thus compare the true value of the upper bound with the proposed estimates, and evaluate the coverage, which is further investigated through a large-scale simulation exercise in Section 8. A nonstandard application is discussed in Section 7, where the distribution of an ordered categorical variable, potentially prone to measurement error, is analyzed. The paper closes with Section 9 providing some concluding remarks. The Supporting Information available at the Biometrics website describes the analytical and bootstrap approaches to calculate the variance for the proposed upper bound estimators; it also provides some further results for the real data and simple $\mathrm{R}$ code to reproduce our results.

\section{2 | MOTIVATION}

Before proceeding to introduce the notation and the technical contents of the paper, we would like to motivate our approach and briefly explain why a practitioner could be interested in using it. Our aim is to propose a way to estimate the maximum size of a hidden population; the justification for this approach stems from the empirical evidence that, in some specific cases, this count may be of major importance.

For example, let us consider the outbreak of hepatitis A virus (HAV) that occurred in and around a college in northern Taiwan from April to July 1995, see Chao (2001) for further details. To quantify the extent of this outbreak, the number of hepatitis cases was determined using an imperfect
TA B L E 1 Benchmark data

$\begin{array}{lrrrrrrrrrr}\text { Dataset } & \boldsymbol{n}_{\mathbf{0}} & \boldsymbol{n}_{\mathbf{1}} & \boldsymbol{n}_{\mathbf{2}} & \boldsymbol{n}_{\mathbf{3}} & \boldsymbol{n}_{\mathbf{4}} & \boldsymbol{n}_{\mathbf{5}} & \boldsymbol{n}_{\mathbf{6}} & \boldsymbol{n}_{\mathbf{7}} & \boldsymbol{n}_{\mathbf{8}} & \boldsymbol{n} \\ \text { Golf } & 88 & 46 & 28 & 21 & 13 & 23 & 14 & 6 & 11 & 162 \\ \text { Sidney } & 22 & 8 & 12 & 16 & 21 & 12 & 31 & & & 100 \\ \text { Cottontail } & 59 & 43 & 16 & 8 & 6 & 0 & 2 & 1 & 76 \\ \text { Taxicab } & 137 & 142 & 81 & 49 & 7 & 3 & 1 & & 283 \\ \text { Hepatitis } & 274 & 187 & 56 & 28 & & & & & 271\end{array}$

Note. Complete data distribution.

screening procedure based on merging three lists (serum test records, hospital records, and epidemiologists' records) of the infected. Twenty-eight students were present in all three lists, 56 in two lists, and 187 in only one list. Therefore, the number of infected students observed was $n=271$. However, some students with hepatitis were missed by all three sources. In fact, a definitive serological test was later performed on all students at the college and a total of $N=545$ students were found to have hepatitis. In other words, $n_{0}=274$ infected students were not recorded by any of the sources. The data are presented in the last row of Table 1. In this case, having an estimate of the maximum extent of the outbreak could be important from the perspective of prevention and healthcare. This may help to plan specific health policy actions and to define the size of the corresponding health-providing services. The same question may arise in the social sciences, when we consider deviant (Böhning et al., 2004) or illegal (van der Heijden et al., 2003; King et al., 2014) behavior; in fact, the estimates we provide may give an idea of the social burden of the problem.

Even when the problem is that of estimating the richness of a population, the maximum number of classes/species could be of interest in itself or worth using to obtain a more precise estimate, based on both lower and upper bounds for such a quantity; see Eren et al. (2012) for an application to archaeology. A similar aim can also be found in software engineering, where the maximum number of defects in a software application is often of interest, see Liu et al. (2015) or, just to give an example, the web page Software Testing Help (2020) containing a discussion of the importance of such information in software testing. In all of these cases, having a reliable estimate of the maximum size of the population of interest could be a crucial step toward providing a timely and successful solution to the problem.

\section{3 | ORDINAL MODELS FOR CAPTURE-RECAPTURE EXPERIMENTS}

Let us start by defining some notation that will be used in the following sections. We start from a target, partially observed, 
population, with the aim of estimating its size, denoted by $N$. For this purpose, we use $m$ identification sources/sampling occasions that register units from the population. We may consider empirical situations where we either use the same mechanism repeatedly on $m$ subsequent occasions, or several different registration sources. The mechanism(s) allow us to observe only a portion of the population. More precisely, we consider a binary indicator variable $x_{i j}$ that equals 1 if the $i$ th unit has been identified by the $j$ th source/sampling occasion, and 0 otherwise. Observed units fulfill the condition $x_{i}=\sum_{j=1}^{m} x_{i j}>0$, whereas the others, having $x_{i}=0$, remain unobserved. The number of sampling occasions, $m$, may be known a priori, or it may be the maximum observed count. Rearranging unit indices, we may denote the global population by $x_{1}, \ldots, x_{N}$ and the observed sample by $x_{1}, \ldots, x_{n}$; in this way, $x_{n+1}=\cdots=x_{N}=0$, without any loss of generality.

The target population can be described by the probability density function $\left(x, \tau_{x}\right)$, where $x=0,1, \ldots, m$, and $\tau_{x}$ denotes the probability that a generic unit from the population is observed exactly $x$ times. Clearly, the usual constraints $\tau_{x} \geq 0$ and $\sum_{x=0}^{m} \tau_{x}=1$ apply. In the following, we will denote the observed frequency distribution by $\left(x, n_{x}\right)$. The size of the observed sample is $n=\sum_{x>0} n_{x}$ and the corresponding distribution is truncated at zero, in the sense that units with $x=0$ are not observed and therefore not in the sample. An obvious estimate for $\tau_{x}$ would be the relative frequency $n_{x} / N$ that, however, cannot be computed as $N$ is unknown. The empirical relative frequency $n_{x} / n$ provides an estimate of the zerotruncated probability $\tau_{x} /\left(1-\tau_{0}\right)$. Due to such a design, $n_{0}$ and $N=\sum_{x=0}^{m} n_{x}$ are both unknown, and finding an estimate for the population size $N$ on the basis of the observed zerotruncated distribution is a special case of the general capturerecapture problem, see Bunge and Fitzpatrick (1993), Wilson and Collins (1992), Chao (2001).

In this framework, the random variable $X_{i}=\sum_{j=1}^{m} X_{i j}$, $X_{i j} \in\{0,1\}$ denotes the number of captures for a given individual; the corresponding values can be considered as the categories of an ordinal random variable. As noted before, the equalities $n=\sum_{i=1}^{N} \llbracket\left(X_{i}>0\right)$ and $n_{0}=N-n=$ $\sum_{i=1}^{N} \llbracket\left(X_{i}=0\right)$ hold by definition, where $\llbracket(a)$ represents the indicator function for the event $a$. We write the complete data cumulative distribution function as

$$
\operatorname{Pr}(X \leq j)=\sum_{x \leq j} \tau_{x}=\pi_{j}=\exp \left(\theta_{j}\right) /\left[1+\exp \left(\theta_{j}\right)\right]
$$

where
Clearly, we have that $\pi_{j} \leq \pi_{j^{\prime}}$ and $\theta_{j} \leq \theta_{j^{\prime}}, j<j^{\prime}=1, \ldots, m$. If we consider the zero-truncated distribution, we may write

$$
\operatorname{Pr}(X \leq j \mid X>0)=p_{j}
$$

and the following equality holds:

$$
\begin{aligned}
\pi_{j} & =\operatorname{Pr}(X \leq j)=\operatorname{Pr}(X=0)+\operatorname{Pr}(0<X \leq j) \\
& =\pi_{0}+\left(1-\pi_{0}\right) p_{j}
\end{aligned}
$$

Solving for $\pi_{0}$, we obtain

$$
\pi_{0}=\frac{\pi_{j}-p_{j}}{1-p_{j}}
$$

or, rather

$$
p_{j}=\frac{\pi_{j}-\pi_{0}}{1-\pi_{0}}
$$

Based on the ordinal nature of $X$, we may write

$$
\theta_{j}=\theta_{0}+\psi_{j}
$$

where

$$
\begin{aligned}
\psi_{j} & =\log \left(\frac{\pi_{j}\left(1-\pi_{0}\right)}{\pi_{0}\left(1-\pi_{j}\right)}\right)=\log \left(\frac{\pi_{j}}{\pi_{0}} \frac{1}{\left(1-p_{j}\right)}\right) \\
& =\log \left(\frac{\pi_{j}}{\pi_{j}-p_{j}}\right)
\end{aligned}
$$

and obtain the following constraints:

$$
\psi_{j} \geq 0, \quad \psi_{j} \leq \psi_{j^{\prime}}, \quad \text { for } j \leq j^{\prime}=1, \ldots, m .
$$

As $\exp \left(\theta_{j}\right)=\exp \left(\theta_{0}+\psi_{j}\right)=\exp \left(\psi_{j}\right) \exp \left(\theta_{0}\right)$, the generic term of the truncated probability distribution $p_{j}$ can be written as

$$
\begin{aligned}
p_{j} & =\frac{\exp \left(\theta_{j}\right)-\exp \left(\theta_{j}-\psi_{j}\right)}{1+\exp \left(\theta_{j}\right)}=\frac{\exp \left(\theta_{j}\right)}{1+\exp \left(\theta_{j}\right)}\left(1-\frac{1}{\exp \left(\psi_{j}\right)}\right) \\
& =\pi_{j}\left(1-\gamma_{j}\right),
\end{aligned}
$$

where the elements $\gamma_{j}=\exp \left(-\psi_{j}\right)=\frac{\exp \left(\theta_{0}\right)}{\theta_{j}}$ fulfill the constraints

$$
1 \geq \gamma_{j} \geq \gamma_{j^{\prime}}, \quad j \leq j^{\prime}=1, \ldots, m \text {. }
$$

We can thus write $\pi_{j}=\frac{p_{j}}{1-\gamma_{j}}$ and obtain the following $m$ equalities for $\pi_{0}$ :

$$
\pi_{0}=\frac{\pi_{j}-p_{j}}{1-p_{j}}=\frac{\gamma_{j} p_{j}}{\left(1-\gamma_{j}\right)\left(1-p_{j}\right)}, \quad j=1, \ldots, m .
$$

Given that the truncated distribution $n_{j}, j=1, \ldots, m$, is observed, providing an estimate for $\pi_{0}$ is equivalent to providing an estimate for $\gamma_{j}$, for a given $j=1, \ldots, m$. However, 
the only information we can derive for $\gamma_{j}$ is that $\gamma_{j} \leq\left(1-p_{j}\right)$, $j=1, \ldots, m$, as $\pi_{0} \leq 1$ in Equation (3), and this is of limited value. In fact, as $\gamma_{j} \rightarrow\left(1-p_{j}\right), \pi_{0} \rightarrow 1$, leading to an uninformative infinite upper bound for the population size.

\section{4 | AN UPPER BOUND FOR $N$}

Our purpose is, however, to develop an upper bound for $N$ that can be readily used in empirical applications. For this purpose, let us consider two indices $j$ and $h$ with $j<h=1, \ldots, m$. We know that the following inequalities hold:

$$
\begin{aligned}
& \pi_{h} \geq \pi_{j}: \\
& p_{h} \geq p_{j} ; \\
& \gamma_{h} \leq \gamma_{j} .
\end{aligned}
$$

Based on Equation (1), we may write

$$
1-p_{j}=1-\frac{\pi_{j}-\pi_{0}}{1-\pi_{0}}=\frac{1-\pi_{0}-\pi_{j}+\pi_{0}}{1-\pi_{0}}=\frac{1-\pi_{j}}{1-\pi_{0}},
$$

and therefore, from Equation (2), we have

$$
\begin{aligned}
\frac{1-p_{j}}{1-p_{h}} & =\frac{1-\pi_{j}}{1-\pi_{h}}=\frac{1-\frac{p_{j}}{1-\gamma_{j}}}{1-\frac{p_{h}}{1-\gamma_{h}}}=\frac{\frac{1-\gamma_{j}-p_{j}}{1-\gamma_{j}}}{\frac{1-\gamma_{h}-p_{h}}{1-\gamma_{h}}} \\
& =\frac{1-\gamma_{j}-p_{j}}{1-\gamma_{h}-p_{h}} \frac{1-\gamma_{h}}{1-\gamma_{j}}=\frac{1-\gamma_{j}-p_{j}}{1-\gamma_{h}-p_{h}} \frac{p_{h}}{\pi_{h}} \frac{\pi_{j}}{p_{j}} \\
& =\frac{1-\gamma_{j}-p_{j}}{1-\gamma_{h}-p_{h}} \frac{p_{h}}{p_{j}} \frac{\pi_{j}}{\pi_{h}} \leq \frac{1-\gamma_{j}-p_{j}}{1-\gamma_{h}-p_{h}} \frac{p_{h}}{p_{j}}
\end{aligned}
$$

as $\pi_{j} \leq \pi_{h}$. The inequality holds for $j<h=1, \ldots,(m-1)$ as for $h=m$ we would have $p_{h}=\pi_{h}=1$ and the ratio in Equation (5) would be infinite. Taking first and last terms of Equation (5) into account, we obtain

$$
\frac{1-p_{j}}{1-p_{h}} \leq\left(\frac{1-\gamma_{j}-p_{j}}{1-\gamma_{h}-p_{h}}\right) \frac{p_{h}}{p_{j}}
$$

or, equivalently

$$
\left(\frac{1-\gamma_{j}-p_{j}}{1-\gamma_{h}-p_{h}}\right) \geq \frac{p_{j}\left(1-p_{j}\right)}{p_{h}\left(1-p_{h}\right)} .
$$

Let us recall the definition for $\gamma_{j}$ :

$$
\begin{aligned}
\gamma_{j} & =\frac{\exp \left(\theta_{0}\right)}{\exp \left(\theta_{j}\right)}=\frac{\exp \left(\theta_{0}\right)}{\exp \left(\theta_{j}\right)} \frac{\exp \left(\theta_{h}\right)}{\exp \left(\theta_{h}\right)}=\frac{\exp \left(\theta_{0}\right)}{\exp \left(\theta_{h}\right)} \frac{\exp \left(\theta_{h}\right)}{\exp \left(\theta_{j}\right)} \\
& =\gamma_{h} \frac{\pi_{h}\left(1-\pi_{j}\right)}{\pi_{j}\left(1-\pi_{h}\right)}=\gamma_{h} \frac{\pi_{h}\left(1-p_{j}\right)}{\pi_{j}\left(1-p_{h}\right)}=\gamma_{h} \frac{1}{\alpha_{j, h}} .
\end{aligned}
$$

This helps us restate the inequality in Equation (7) as follows:

$$
\begin{aligned}
& \left(1-\gamma_{j}-p_{j}\right) \geq\left(1-\gamma_{h}-p_{h}\right) \frac{p_{j}\left(1-p_{j}\right)}{p_{h}\left(1-p_{h}\right)} \\
& =\left(1-\gamma_{j} \alpha_{j, h}-p_{h}\right) \frac{p_{j}\left(1-p_{j}\right)}{p_{h}\left(1-p_{h}\right)} .
\end{aligned}
$$

Simplifying the inequality in Equation (8) gives

$$
\begin{gathered}
\gamma_{j}\left[1-\alpha_{j, h} \frac{p_{j}\left(1-p_{j}\right)}{p_{h}\left(1-p_{h}\right)}\right] \leq\left(1-p_{j}\right)-\left(1-p_{h}\right) \frac{p_{j}\left(1-p_{j}\right)}{p_{h}\left(1-p_{h}\right)} \\
\quad=\left(1-p_{j}\right)-\frac{p_{j}\left(1-p_{j}\right)}{p_{h}}=\frac{\left(p_{h}-p_{j}\right)\left(1-p_{j}\right)}{p_{h}} .
\end{gathered}
$$

Let us recall that

$$
\begin{aligned}
\gamma_{j}\left[1-\alpha_{j, h} \frac{p_{j}\left(1-p_{j}\right)}{p_{h}\left(1-p_{h}\right)}\right] & =\gamma_{j}\left[1-\frac{\pi_{j}\left(1-p_{h}\right)}{\pi_{h}\left(1-p_{j}\right)} \frac{p_{j}\left(1-p_{j}\right)}{p_{h}\left(1-p_{h}\right)}\right] \\
& =\gamma_{j}\left[1-\frac{\pi_{j}}{\pi_{h}} \frac{p_{j}}{p_{h}}\right] .
\end{aligned}
$$

It is straightforward to show that the simplified term in the square brackets $\left(1-\frac{\pi_{j}}{\pi_{h}} \frac{p_{j}}{p_{h}}\right)$ is positive; solving for $\gamma_{j}, j<h=$ $1, \ldots,(m-1)$, we obtain the upper bound we have been looking for:

$$
\gamma_{j} \leq \frac{\left(p_{h}-p_{j}\right)\left(1-p_{j}\right)}{p_{h}\left[1-\frac{\pi_{j}}{\pi_{h}} \frac{p_{j}}{p_{h}}\right]}=\left(1-p_{j}\right)\left(\frac{p_{h}-p_{j}}{p_{h}-p_{j} \frac{\pi_{j}}{\pi_{h}}}\right)=\gamma_{j}^{u}
$$

for any $j$ and $h$ such that $j<h=1, \ldots,(m-1)$. It is worth noting that as $\pi_{j} \leq \pi_{h}$ for $j<h=1, \ldots,(m-1)$, we have that $\gamma_{j}^{u} \leq\left(1-p_{j}\right)$, where the term $\left(1-p_{j}\right)$ defines a trivial bound for $\gamma_{j}$, as we have discussed previously. Recalling Equation (3), an upper bound is also obtained for $\pi_{0}$ :

$$
\begin{aligned}
\pi_{0} & =\frac{\pi_{j}-p_{j}}{1-p_{j}}=\frac{\gamma_{j} p_{j}}{\left(1-\gamma_{j}\right)\left(1-p_{j}\right)} \leq \frac{\gamma_{j}^{u} p_{j}}{\left(1-\gamma_{j}^{u}\right)\left(1-p_{j}\right)} \\
& =\pi_{0 j}^{u}, \quad j=1, \ldots, m
\end{aligned}
$$

and for the population size:

$$
N_{(j)}^{u}=\frac{n}{1-\pi_{0 j}^{u}}=\frac{n}{1-\left[\frac{\gamma_{j}^{u}}{1-\gamma_{j}^{u}} \frac{p_{j}}{1-p_{j}}\right]} .
$$

\section{5 | AN APPROXIMATION}

Unfortunately, $\gamma_{j}^{u}$ cannot be computed as the ratio $\frac{\pi_{j}}{\pi_{h}}$ is unknown, as is $\pi_{0}$. For one of the elements in the ratio, say $\pi_{h}$, we may use the fact that if $h=m, \pi_{m}=1$. But, as we 
have seen before, in this case $\gamma_{j}^{u}$ is undefined. To get around this, we can approximate $\gamma_{j}^{u}$ using the data at hand, which is the observed truncated distribution. The first approximation comes from considering that

$$
\frac{p_{j}}{p_{h}} \simeq \frac{\pi_{j}}{\pi_{h}}
$$

leading to

$$
\gamma_{j} \simeq \gamma_{j}^{\star}=\left(1-p_{j}\right)\left(\frac{p_{h}-p_{j}}{p_{h}-p_{j} \frac{p_{j}}{p_{h}}}\right) .
$$

To get a good approximation, however, one of two conditions should be met: either $\pi_{0}$ is close to 0 or, if this is not the case, $j$ and $h$ should be large enough to ensure that $\pi_{h}>\pi_{j} \gg \pi_{0}$. In other words, we should look at higher categories (eg, counts). In particular, due to the problems with setting $h=m$ that we have already outlined, we suggest to set $h=m-1$ and $j=$ $h-1=m-2$. In this case,

$$
\gamma_{(m-2)}^{\star}=\frac{p_{m-1}}{\left(p_{m-1}+p_{m-2}\right)}\left(1-p_{m-2}\right) .
$$

Obviously, we have that

$\frac{\pi_{(m-2)}}{\pi_{(m-1)}}=\frac{p_{(m-2)}}{1-\gamma_{(m-2)}} \frac{1-\gamma_{(m-1)}}{p_{(m-1)}}=\frac{p_{(m-2)}}{p_{(m-1)}} \frac{1-\gamma_{(m-1)}}{1-\gamma_{(m-2)}} \geq \frac{p_{(m-2)}}{p_{(m-1)}}$

as $\gamma_{(m-2)} \geq \gamma_{(m-1)}$. The equality holds either when $\pi_{0}=0$ or $p_{(m-2)}=p_{(m-1)}$, that is $\pi_{(m-2)}=\pi_{(m-1)}$, which, however, leads to the trivial bound $\gamma_{(m-2)}^{\star}=\left(1-p_{(m-2)}\right)$. According to the previous inequality, in the general case, it holds that

$$
\gamma_{(m-2)}^{u}>\gamma_{(m-2)}^{\star} .
$$

Using $\gamma_{(m-2)}^{\star}$ instead of $\gamma_{(m-2)}^{u}$, we obtain the following approximation for the upper bound in Equation (11):

$$
\begin{aligned}
N_{(m-2)}^{u} & =N^{u}=\frac{n}{1-\pi_{0}(m-2)} \simeq N_{\star}^{u} \\
& =\frac{n}{1-\left[\frac{\gamma_{(m-2)}^{\star}}{1-\gamma_{(m-2)}^{\star}} \frac{p_{(m-2)}}{1-p_{(m-2)}}\right]}=\frac{n}{1-\left(\frac{p_{(m-1)}}{1+p_{(m-1)}}\right)} .
\end{aligned}
$$

This estimator has a remarkably simple structure and is easy to obtain; if we look at the observed frequency distribution $\left(x, n_{x}\right), x=1, \ldots, m$ and use $\hat{p}_{j}=\frac{\sum_{l \leq j} n_{l}}{n}, j=1, \ldots, m$, we get the estimate:

$$
\begin{aligned}
\hat{N}_{\star}^{u} & =\frac{n}{1-\left(\frac{\hat{p}_{(m-1)}}{1+\hat{p}_{(m-1)}}\right)}=n\left(1-\hat{p}_{(m-1)}\right) \\
& =n+\sum_{j=1}^{(m-1)} n_{j}=2 n-n_{m} .
\end{aligned}
$$

A sufficient condition for $N_{\star}^{u}$ to be an upper bound for $N$ is that $\gamma_{(m-2)}^{\star} \geq \gamma_{(m-2)}$, that is

$$
\begin{aligned}
\gamma_{(m-2)}^{\star} & =\frac{p_{(m-1)}}{p_{(m-1)}+p_{(m-2)}}\left(1-p_{(m-2)}\right) \\
& \geq 1-\frac{p_{(m-2)}}{\pi_{(m-2)}}=\gamma_{(m-2)} .
\end{aligned}
$$

If we solve for $\pi_{(m-2)}$, we obtain the following condition for $N_{\star}^{u}$ to be an upper bound of the true population size, $N$ :

$$
\pi_{(m-2)} \leq \frac{p_{(m-1)}+p_{(m-2)}}{p_{(m-1)}+1} .
$$

By exploiting the dependence of $\pi_{(m-2)}$ on $\pi_{0}$ and $p_{(m-2)}$, we may also derive a condition on $\pi_{0}$ for $N_{\star}^{u}$ to be an upper bound of $N$ :

$$
\pi_{0} \leq \frac{p_{(m-1)}}{1+p_{(m-1)}} \simeq 0.5 .
$$

However, this sufficient condition cannot be fulfilled in every situation, as the probability of zero counts could be higher than 0.5 , and/or the number of capture occasions could be very small (eg, three), leading to a substantial difficulty in the use of $p_{(m-2)} / p_{(m-1)}$ to approximate the ratio $\pi_{(m-2)} / \pi_{(m-1)}$. More importantly, no method can be used to check (empirically) that the condition in Equation (18) holds. Therefore, we introduce a further upper bound approximate estimator, which we will refer to as $N_{c}^{u}$.

This is simply based on estimating $\pi_{0}$ from the observed distribution, plugging in the estimate to obtain an estimate for $\pi_{j}, j=1, \ldots, m-1$, and deriving the upper bound based on this augmented empirical distribution.

For this purpose, we use the Chao's (1984) lower bound estimator to approximate the number of missing units $n_{0}$ :

$$
\widehat{n}_{0}^{c}=\frac{n_{1}^{2}}{2 n_{2}}
$$

or, equivalently

$$
\widehat{\pi}_{0}^{c}=\frac{\widehat{n}_{0}^{c}}{\widehat{n}_{0}^{c}+n} .
$$

Based on such an estimator, we derive a completed distribution $\left(\widehat{n}_{0}^{c}, n_{1}, \ldots, n_{m}\right)$ and calculate the upper bound for $N$ on such a completed distribution. Clearly, a different estimator for $n_{0}$ can be used, such as those described in Lanumteang and Böhning (2011) or Rocchetti et al. (2014), as the procedure does not depend on the specific form of the estimator. The approach we propose leads to the following values for the cumulative probabilities of the completed data:

$$
\hat{\pi}_{j}^{c}=\hat{\pi}_{0}^{c}+\left(1-\hat{\pi}_{0}^{c}\right) p_{j}, \quad j=1, \ldots,(m-1) .
$$


The approximation for $\gamma_{(m-2)}^{u}$ obviously follows:

$$
\gamma_{(m-2)}^{c}=\left(1-p_{(m-2)}\right)\left(\frac{p_{(m-1)}-p_{(m-2)}}{p_{(m-1)}-p_{(m-2)} \frac{\hat{\pi}_{(m-2)}^{c}}{\hat{\pi}_{(m-1)}^{c}}}\right)
$$

and the approximation to the upper bound follows from Equation (11):

$$
N_{c}^{u}=N_{(m-2), c}^{u}=\frac{n}{1-\left[\frac{\gamma_{(m-2)}^{c}}{1-\gamma_{(m-2)}^{c}} \frac{p_{(m-2)}}{1-p_{(m-2)}}\right]} .
$$

As we have mentioned previously, we know that $N \leq N_{c}^{u}$ whenever $\gamma_{(m-2)} \leq \gamma_{(m-2)}^{c}$. Starting from this inequality, we obtain the following condition for $N_{c}^{u}$ to be an upper bound for the true population size, $N$ :

$$
\begin{aligned}
& \pi_{(m-2)} \\
& \quad \leq p_{(m-2)}\left[1-\left(1-p_{(m-2)}\right)\left(\frac{p_{(m-1)}-p_{(m-2)}}{p_{(m-1)}-p_{(m-1)} \frac{\hat{\pi}_{(m-2)}^{c}}{\hat{\pi}_{(m-1)}^{c}}}\right)\right]^{-1}
\end{aligned}
$$

or, by doing a little algebra, the equivalent condition

$$
\pi_{0} \leq \frac{\left(p_{(m-1)}-p_{(m-2)}\right)}{\left[\left(1-\frac{\hat{\pi}_{(m-2)}^{c}}{\hat{\pi}_{(m-1)}^{c}}\right)+\left(p_{(m-1)}-p_{(m-2)}\right)\right]} .
$$

That is, with an increasing number of capture occasions, $m$, and/or an increasing value of the ratio $\frac{\hat{\pi}_{(m-2)}^{c}}{\hat{\pi}_{(m-1)}^{c}}$, the proposed estimator gives a reliable upper bound for $N$.

\section{6 | REAL DATA EXAMPLES}

In this section, we provide a re-analysis of five well-known benchmark datasets. For each of these, the global population size $N$, as well as $n_{0}$, are known; this is the reason for selecting them, as we aim to compare the approximate upper bound estimate and the true population size. For each of these studies, we calculate the exact value for $N^{u}$, the upper bound estimate in (11), and compare it with the estimates $\hat{N}_{\star}^{u}$ and $\widehat{N}_{c}^{u}$, also looking at the difference between $p_{(m-1)} / p_{(m-2)}$ and $\pi_{(m-1)} / \pi_{(m-2)}$. We also provide the analytical and bootstrap estimates for the standard deviation of $\hat{n}_{0 \star}^{u}=\widehat{N}_{\star}^{u}-n$ and $\widehat{n}_{0 c}^{u}=\widehat{N}_{c}^{u}-n$. These are calculated using the procedure described in the Supporting Information, available at the Biometrics website.

\section{1 | Golf Tees data}

The 1999 statistics honors class at the University of St. Andrews (Scotland) participated in the following experiment, see Borchers et al. (2004). A set of 760 golf tees of two different colors were arranged into 250 groups of various sizes, which were placed in a survey region of $1680 \mathrm{~m}^{2}$, either exposed above the surrounding grass, or partly hidden by it. A total of 162 groups of tees were found by the participants while $n_{0}=88$ were not. The truncated empirical distribution (see the first row of Table 1) refers to the number of times each group of tees was found, with $m=8$ sources corresponding to eight independent observers. The approximate estimates of the upper bound for the population size are $\hat{N}_{\star}^{u}=313$ and $\hat{N}_{c}^{u}=350$; these are both reasonable values for $N=250$, as well as good approximations for $N^{u}=401$. The bootstrap estimate for the standard deviation of $\widehat{n}_{0 \star}^{u}$ is $b . s d\left(\widehat{n}_{0 \star}^{u}\right)=8.9$, whereas the analytical estimate is higher, $\operatorname{a.s} d\left(\widehat{n}_{0 \star}^{u}\right)=10.9$. The bootstrap estimate for the standard deviation of $\widehat{n}_{0 c}^{u}, b . s d\left(\widehat{n}_{0 c}^{u}\right)=23.08$, is considerably higher than the former as it likely suffers from the additional variability in Chao's estimator $\widehat{n}_{0}^{c}$. We also note that the truncated and untruncated probability ratios for counts $(m-2)$ and $(m-1)$ are very close to each other $(0.96$ and 0.975 , respectively).

\subsection{Sidney data}

The data concern a screening test for bowel cancer, the fecal occult blood test: this can be used to detect the presence of a small amount of blood in the bowel motion as an indicator for cancer prior to the manifestation of clear symptoms. From 1984 onward, about 50000 subjects were screened for bowel cancer at St Vincent's Hospital in Sydney (Australia), see Lloyd and Frommer (2004a, 2004b, 2008). Given that a single application of the test is not sufficiently accurate, the screening procedure was based on a sequence of $m=6$ binary diagnostic tests performed on consecutive days; for each test, the presence $(x=1)$ or the absence $(x=0)$ of blood in feces was recorded. People with negative results in all six tests did not undergo further assessment and their true disease status remained unknown, as this can only be ascertained by carrying out a definitive diagnostic test. People with at least one positive test result had their true disease status verified by physical examination, sigmoidoscopy, and colonoscopy. A sample of $N=122$ individuals with confirmed bowel cancer have been screened again using the same procedure and the same number of testing occasions $(m=6)$. The corresponding distribution is reported in the second row of Table 1. The approximate estimates of the upper bound for the population size, $\widehat{N}_{\star}^{u}=169$ and $\widehat{N}_{c}^{u}=172$, are quite close to each other and to the true value $N^{u}=191$, see the second row 
TA B L E 2 Benchmark data

\begin{tabular}{|c|c|c|c|c|c|c|c|c|c|}
\hline Dataset & $N$ & $\widehat{N}_{\star}^{u}$ & $\widehat{N}_{c}^{u}$ & $N^{u}$ & $\operatorname{b.sd}\left(\hat{n}_{0 \star}^{u}\right)$ & $\operatorname{a.sd}\left(\hat{n}_{0 \star}^{u}\right)$ & b.sd $\left(\hat{n}_{0 c}^{u}\right)$ & $\frac{p_{(m-2)}}{p_{(m-1)}}$ & $\frac{\pi_{(m-2)}}{\pi_{(m-1)}}$ \\
\hline Golf & 250 & 313 & 350 & 401 & 8.92 & 10.91 & 23.08 & 0.96 & 0.975 \\
\hline Sidney & 122 & 169 & 172 & 191 & 6.41 & 7.26 & 14.94 & 0.826 & 0.868 \\
\hline Taxicab & 420 & 565 & 691 & 702 & 12.02 & 13.21 & 34.42 & 0.989 & 0.993 \\
\hline Hepatitis & 545 & 543 & 827 & 817 & 11.77 & 12.45 & 23.16 & 0.897 & 0.949 \\
\hline
\end{tabular}

Note. Upper bound estimates for population size $N$.

of Table 2. The bootstrap estimate for the standard deviation is $b . s d\left(\hat{n}_{0 \star}^{u}\right)=6.41$, whereas the analytical estimate, $\operatorname{a.sd}\left(\hat{n}_{0 \star}^{u}\right)=7.26$, is higher. As in Section 6.1, the bootstrap estimate for $\hat{n}_{0 c}^{u}$ is higher than the other two, $\operatorname{b.s} d\left(\hat{n}_{0 c}^{u}\right)=$ 14.94. The ratios of the truncated and untruncated probabilities for the counts $(m-2)$ and $(m-1)$ are equal to 0.83 and 0.87 , respectively.

\section{3 | Cottontail data}

A live trapping study of a cottontail rabbit population with known size $N=135$, confined within a 4-acre zone, was conducted for $m=18$ consecutive nights by Edwards and Eberhardt (1967). This dataset has been studied extensively, starting from the seminal paper by Chao (1987). Here, the number of trapping occasions is $m=18$ but the maximum count is $\widetilde{m}=7$, see the third row of Table 1 .

The approximate estimates of the upper bound for the population size are $\hat{N}_{\star}^{u}=151$ and $\hat{N}_{c}^{u}=212$; the latter is essentially equal to the true value $N^{u}=210$. The bootstrap estimate of the standard deviation for $\widehat{n}_{0 \star}^{u}$ is equal to $b . s d\left(\hat{n}_{0 \star}^{u}\right)=$ 6.03 , the analytical estimate is slightly higher, $\operatorname{a.s} d\left(\hat{n}_{0 \star}^{u}\right)=$ 6.99 , and $b . s d\left(\hat{n}_{0 c}^{u}\right)=16.41$ is, as we have seen before, much higher than both the previous estimates. The ratios of the truncated and untruncated probabilities for the counts $(m-2)$ and $(m-1)$ are equal to 0.97 and 0.98 , respectively.

\section{4 | Taxicab data}

Carothers (1973) conducted an experiment on the taxicab population of Edinburgh, Scotland. In this example, the population has a known size $N=420$; the experiment roughly consisted of a capture when a taxicab was sighted. The taxicab population was observed on $m=10$ consecutive days, with observation points and times changing with each day; however, no taxis were observed more than $\widetilde{m}=6$ times, see the fourth row of Table 1.

In this case, $N^{u}=702$ is much higher than the proposed approximation $\widehat{N}_{\star}^{u}=565$, but it is well approximated by $\widehat{N}_{c}^{u}=691$. Both approximate estimates are, however, quite successful given that $N=420$. The ratios of the truncated and the untruncated probabilities for the counts $(m-2)$ and $(m-1)$ are equal to two decimal places with a value of 0.99 .

\section{5 | Hepatitis data}

Now we return to the hepatitis data introduced in Section 2. As we mentioned previously, the data refer to an outbreak of the HAV recorded in and around a college in northern Taiwan from April to July 1995, see Chao (2001). Three sources were merged and the corresponding zero-truncated distribution is reported in the last row of Table 1. To estimate the extent of the outbreak, a screening serological check for all students was conducted, so that the true population size $N=545$ is known. The proposed approximate estimates are $\widehat{N}_{\star}^{u}=543$ and $\widehat{N}_{c}^{u}=827$. Although the former fails in providing an upper bound for $N$, likely due to the small number of capture occasions considered and the condition $n_{0}<n$ not being fulfilled, the latter satisfactorily approximates the true value of the bound $N^{u}=817$. The ratios of the truncated and untruncated probabilities, provided in Table 1 , are equal to 0.9 and 0.95 , respectively.

\section{6 | Summarizing the empirical findings}

Based on the results we have just discussed, the approximate estimate $\hat{N}_{\star}^{u}$ may fail in two cases: when dealing with a very low number of capture occasions and/or when the number of unseen units is greater than $50 \%$ of the population size, see the condition in Equation (18). We face this issue empirically in the hepatitis example, see Section 6.5, where only three capture occasions and a high proportion of unseen units were available, and in the simulation study, see Section 8, which includes different types of "true" data-generating processes. However, even in these extreme cases, we are still able to rely on $\widehat{N}_{c}^{u}$, with the (negligible) further effort of estimating $\widehat{n}_{0 c}$ and completing the observed data.

Table 2 summarizes the results obtained by calculating the proposed upper bound for each of the five benchmark datasets we considered. We report the following quantities: $\hat{N}_{\star}^{u}$, the 
Golf data

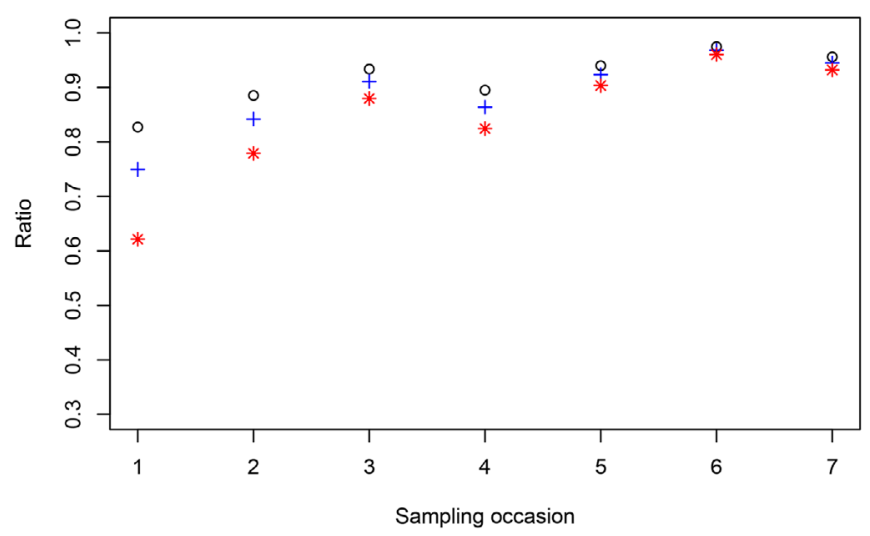

Cottontail data

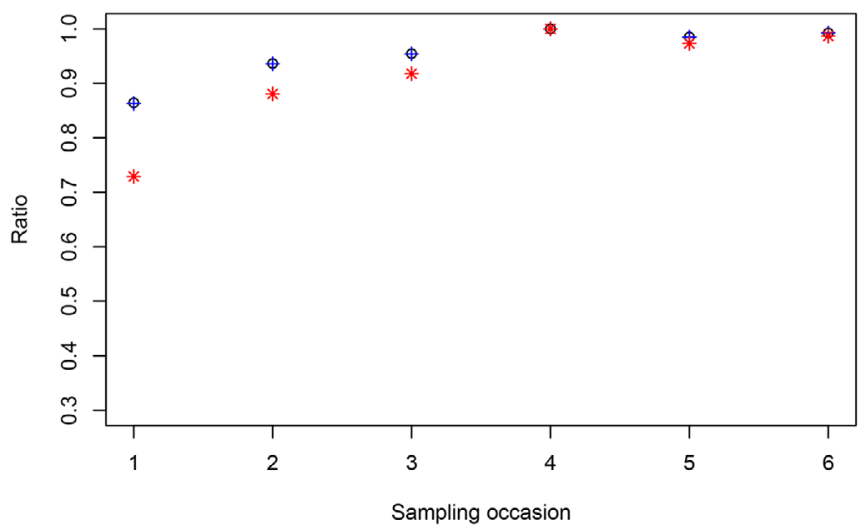

Sidney data

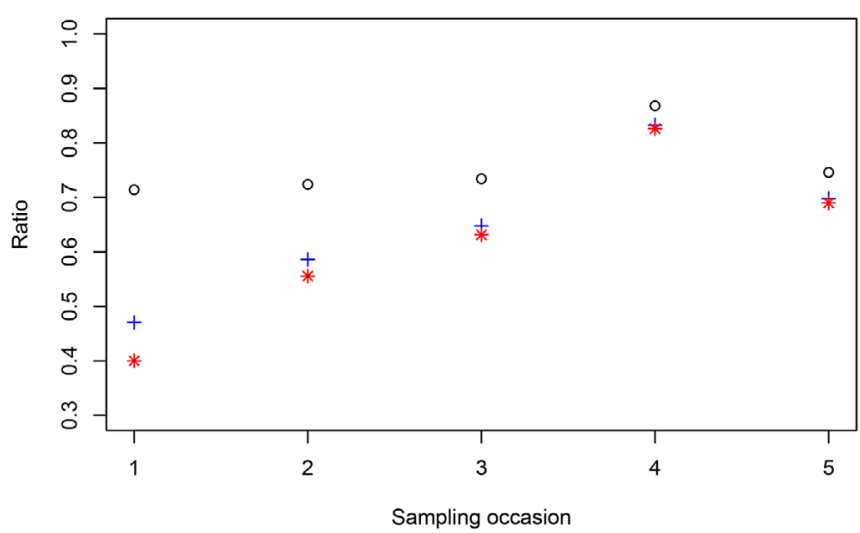

Taxicab data

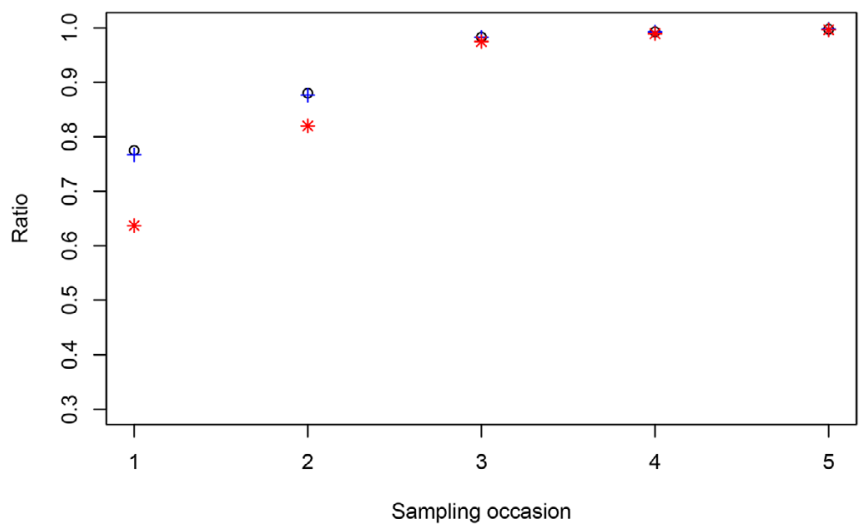

F I G U R E 1 Benchmark data examples. Successive frequency ratios for the truncated (red stars), completed (blue pluses), and untruncated (black dots) distributions. This figure appears in color in the electronic version of the article, and any mention of color refers to that version

TA B L E 3 Oman accident data

\begin{tabular}{|lllllll|}
\hline Gender & No injury & Mild & Moderate & Severe & Fatal & Number of crashes \\
\hline Total & 6790 & 13346 & 9758 & 2226 & 3665 & 35785 \\
\hline Male $(\%)$ & 19.9 & 35.8 & 27.0 & 6.5 & 10.9 & 31763 \\
\hline Female (\%) & 11.9 & 49.3 & 29.6 & 4.0 & 5.1 & 4022 \\
\hline
\end{tabular}

Note. Complete data distribution.

approximate estimate of the upper bound for $N$ given in Equation $11 ; \widehat{N}_{c}^{u}$, the approximate estimate of the upper bound calculated by completing the observed distribution with $\widehat{n}_{0}^{c}$, the Chao estimate for $n_{0} ; N^{u}$, the true value of the upper bound for $N$; and the ratios $p_{(m-2)} / p_{(m-1)}$ and $\pi_{(m-2)} / \pi_{(m-1)}$ to give an idea of the approximation order. We also report the estimates of the standard deviation for $\hat{n}_{0 \star}^{u}$ and $\hat{n}_{0 c}^{u}$. The former has been calculated analytically (a.) and via nonparametric bootstrap (b.), while the bootstrap approach is the only option for the latter, as detailed in Section 1 of the Supporting Information available at the Biometrics website. In Figure 1, we report, excluding the hepatitis data where $m=3$, the ratios $p_{(j)} / p_{(j+1)}$ and $\pi_{(j)} / \pi_{(j+1)}$ for $j=1, \ldots,(m-2)$, to further explore the differences we may observe, in empirical cases, between the truncated and untruncated distributions.

\section{7 | A FURTHER, NONSTANDARD, EXAMPLE}

Up to this point, we have presented the proposed upper bound in the context of a partially observed count distribution; however, it can also be fruitfully applied to truncated distributions involving ordinal categorical variables. The following example may suggest an alternative use of the proposed approach. According to Al Aamri (2018), a total of 35785 road traffic 
TA B L E 4 Oman accident data

\begin{tabular}{|c|c|c|c|c|c|c|c|c|c|}
\hline Dataset & $\widetilde{N}$ & $\widehat{N}_{\star}^{u}$ & $\widehat{N}_{c}^{u}$ & $\tilde{N}^{u}$ & b.sd $\left(\hat{n}_{0 \star}^{u}\right)$ & $\operatorname{a.sd}\left(\hat{\boldsymbol{n}}_{0 \star}^{u}\right)$ & b.sd $\left(\hat{\boldsymbol{n}}_{0 c}^{u}\right)$ & $\frac{p_{(m-2)}}{p_{(m-1)}}$ & $\frac{\pi_{(m-2)}}{\pi_{(m-1)}}$ \\
\hline Crash & 35785 & 54325 & 63450 & 61115 & 118.49 & 120.88 & 267.47 & 0.912 & 0.931 \\
\hline Crash(male) & 31763 & 47440 & 54969 & 53753 & 110.27 & 113.07 & 244.35 & 0.906 & 0.927 \\
\hline
\end{tabular}

Note. Upper bound estimates for population size $N$.

T A B L E 5 Mixing distributions for individual capture probabilities

Mixing distribution

\begin{tabular}{|c|c|c|c|c|c|}
\hline Group A & Details & $\mu$ & $\sigma^{2}$ & skew & $\pi_{0}$ \\
\hline A1. Beta & $B(1.76,9.99)$ & 0.15 & 0.010 & 1.02 & 0.448 \\
\hline A2. Two-point & $\pi=(0.942,0.058), \theta=(0.125,0.552)$ & 0.15 & 0.010 & 3.78 & 0.422 \\
\hline A3. Two-point & $\pi=(0.5,0.5), \theta=(0.05,0.25)$ & 0.15 & 0.010 & 0.00 & 0.457 \\
\hline A4. Two-point & $\pi=(0.964,0.036), \theta=(0.131,0.669)$ & 0.15 & 0.010 & 5.00 & 0.415 \\
\hline A5. Four-point & $\pi=(0.4,0.1,0.1,0.4), \theta=(0.05,0.1,0.2,0.25)$ & 0.15 & 0.009 & 0.00 & 0.445 \\
\hline A6. Uniform & $a=0, b=0.3$ on $[0, b]$ & 0.15 & 0.010 & 0.00 & 0.435 \\
\hline \multicolumn{6}{|l|}{ Group B } \\
\hline B1. Beta & $B(1.31,3.94)$ & 0.25 & 0.030 & 0.80 & 0.306 \\
\hline B2. Two-point & $\pi=(0.866,0.134), \theta=(0.182,0.690)$ & 0.25 & 0.030 & 2.14 & 0.259 \\
\hline B3. Two-point & $\pi=(0.5,0.5), \theta=(0.077,0.423)$ & 0.25 & 0.030 & 0.00 & 0.329 \\
\hline B4. Two-point & $\pi=(0.916,0.084), \theta=(0.198,0.822)$ & 0.25 & 0.030 & 3.00 & 0.242 \\
\hline B5. Four-point & $\pi=(0.4,0.1,0.1,0.4), \theta=(0.06,0.2,0.3,0.44)$ & 0.25 & 0.029 & 0.00 & 0.324 \\
\hline B6. Quadratic & $f_{x}=85.7(x-0.4)^{2}$ on $(0.1,0.6)$ & 0.26 & 0.030 & 1.04 & 0.265 \\
\hline \multicolumn{6}{|l|}{ Group C } \\
\hline C1. Beta & $B(0.49,2.76)$ & 0.15 & 0.030 & 1.54 & 0.550 \\
\hline C2. Two-point & $\pi=(0.935,0.065), \theta=(0.104,0.807)$ & 0.15 & 0.030 & 3.53 & 0.441 \\
\hline C3. Exponential & $\lambda=6$, truncated to $(0,1]$ & 0.16 & 0.030 & 1.68 & 0.479 \\
\hline C4. Log & $f_{x}=-\log (x)$ on $(0,1]$ & 0.25 & 0.050 & 0.89 & 0.371 \\
\hline C5. Beta mix & $\pi=(0.5,0.5), B(0.43,8.08)$ and $B(9.13,27.38)$ & 0.15 & 0.015 & 0.27 & 0.492 \\
\hline C6. Beta mix & $\pi=(0.5,0.5), B(0.81,4.57)$ and $B(3.63,6.74)$ & 0.25 & 0.030 & 0.48 & 0.315 \\
\hline
\end{tabular}

Note. Mean $(\mu)$, variance $\left(\sigma^{2}\right)$ and skewness coefficient of the mixing distributions, $\pi=$ component weight, $\theta=$ component-specific parameter, from Pledger (2005).

crashes were reported in Oman in 2015; $71 \%$ of them resulted in injuries, whereas $10 \%$ were fatal. Table 3 details the frequencies and percentages, stratified by gender.

As the burden of mortality and disability has considerable economic, social, and healthcare implications, it could be of interest to estimate the number of crashes resulting in $n o$ injuries, as the corresponding count could be severely downward biased. In fact, not all of the crashes have been reported, as those with minor consequences are likely to be underreported. That is, the corresponding frequency $\left(n_{0}\right.$ in a capturerecapture setting) may be considered as observed with error; the aim here is to recover this quantity by looking at the zerotruncated (ie, truncated at the no injury category) distribution. We report in Table 4 the approximate estimates $\widehat{N}_{\star}^{u}$ and $\widehat{N}_{c}^{u}$, the latter obtained by completing the observed distribution with the Chao estimate $\widehat{n}_{0}^{c}$ and $N^{u}$. Further, we give the naive, likely underestimated, population size $\tilde{N}$ and the correspond- ing value of the upper bound $\widetilde{N}^{u}$ that is likely underestimated as well. The ratios $\hat{p}_{(m-2)} / \widehat{p}_{(m-1)}$ and $\hat{\pi}_{(m-2)} / \widehat{\pi}_{(m-1)}$ are also reported to give an idea of the approximation order. We further provide the standard deviation estimates for $\widehat{n}_{0 \star}^{u}$ and $\widehat{n}_{0 c}^{u}$, the approximate estimates of the upper bound for $n_{0}$. As before, the former has been calculated both analytically (a.) and via a nonparametric bootstrap (b.) approach, whereas the latter has been calculated by nonparametric bootstrap only.

\section{8 | SIMULATION STUDY}

Pledger (2005) describes a simulation experiment concerning mixed binomial distributions, where several prior distributions for individual-specific detection probabilities are considered. The aim is to give a comprehensive assessment of bias and precision for a class of population size estimators. 
TA B L E 6 Simulation study

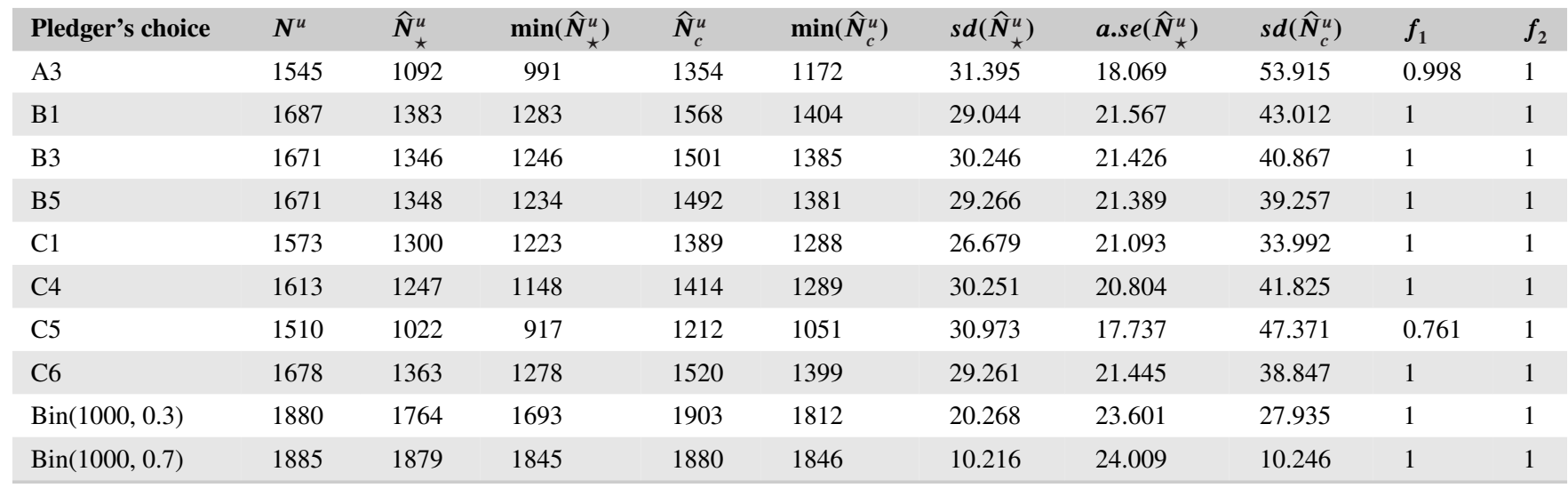

Note. $s d(\cdot)$ and $a . s e(\cdot)$ denote the standard deviation of the estimator across simulations, and the mean of the analytical standard error, respectively. All quantities are averages over simulation samples, $\min (\cdot)$ refers to the minimum over simulation samples; $f_{1}=f\left(\hat{N}_{\star}^{u}>N\right)$ and $f_{2}=f\left(\hat{N}_{c}^{u}>N\right)$ are the relative frequencies for the approximate upper bound being greater than the true population size. In all cases $N=1000$.

The scheme is reported in Table 5. Rocchetti et al. (2014) have presented a point estimator for the size of the population, using this simulation scheme as a benchmark. We propose to use again the same simulation scheme and, in particular, those scenarios where the population size was more difficult to recover, and a substantial underestimation of the true population size was recorded. The study is based on $B=1000$ replications (samples) drawn from different mixed binomial distributions with population size $N=1000$ and $m=6$ sampling occasions.

Table 5 shows the mixing distributions in the Pledger scheme divided into three groups: the first (A) is characterized by a low mean and heterogeneity and a probability of having a zero-count $\left(\pi_{0}\right)$ slightly higher than 0.4 . The second group (B) refers to a situation with a higher mean and heterogeneity, but a smaller mass at zero; group $\mathrm{C}$ is an intermediate situation between $\mathrm{A}$ and $\mathrm{B}$, where the mixing distributions have a low mean but a high heterogeneity and a large mass at zero.

We consider one choice from the first group (A3), three choices from the second group (B1, B3, B5), and four from the third $(\mathrm{C} 1, \mathrm{C} 4, \mathrm{C} 5, \mathrm{C} 6)$; to get an idea of the behavior of the proposed estimator under complete homogeneity conditions, we have also drawn counts from a binomial distribution with common probability of observing a generic unit equal to 0.3 and 0.7 , respectively. Table 6 shows the upper bound for $N$ that we would get if the complete distribution were known $\left(N^{u}\right)$, the mean (across simulations) of the proposed approximate estimates $\left(\hat{N}_{\star}^{u}\right.$ and $\left.\hat{N}_{c}^{u}\right)$, the corresponding minimum values obtained in the 1000 replicates $\left(\min \left(\hat{N}_{\star}^{u}\right)\right.$ and $\left.\min \left(\widehat{N}_{c}^{u}\right)\right)$, the standard deviation of the estimates across simulations $\left(s d\left(\hat{N}_{\star}^{u}\right)\right.$ and $\left.s d\left(\hat{N}_{c}^{u}\right)\right)$, the mean of the analytical standard error $\left(\operatorname{se.} a\left(\hat{N}_{\star}^{u}\right)\right)$, the true $n_{0}$, and the mean estimates $\hat{n}_{\star}^{u}$ and $\widehat{n}_{c}^{u}$. Finally, we report the observed coverage frequency across simulations for $\hat{N}_{\star}^{u}$ and $\widehat{N}_{c}^{u}$, computed as the propor- tion of samples for which the approximate estimates of the upper bound are greater than the true population size $N$.

Looking at the results in Table 6, we notice that the proposed approximate estimator of the upper bound $\widehat{N}_{\star}^{u}$ always performs well in both the heterogeneous and homogeneous settings: its minimum value across replications is always greater than the true $N$ except for choices A3 and C5, where the coverage frequency is slightly less than 1 (0.998 and 0.761, respectively). As a matter of fact, these choices (A3 and C5) correspond to a very high percentage of unseen units, leading to moderate underestimation of the true upper bound. In all cases, the estimator $\hat{N}_{c}^{u}$ gives a reliable approximation to $N^{u}$ and its coverage is always equal to 1 ; consequently, it may be used as a reliable estimator for the upper bound, regardless of the true data generating distribution.

\section{9 | CONCLUDING REMARKS}

Capture-recapture studies have been frequently used to estimate the size of a partially observed population; several approaches based either on semiparametric, for example, finite mixtures, see Pledger (2005), or parametric, for example, negative or beta binomial, see Rocchetti et al. (2011), count distribution models have been used in such a context. Starting by considering a frequency of frequencies distribution and simply exploiting the ordinal nature of the observed counts, we develop a potentially useful upper bound for the population size, which seems to be a novel and undeveloped area in the capture-recapture literature as the obvious upper bound is usually infinite. As the upper bound depends on unobservable terms, we propose two approximate estimators; we study their behavior by using a series of benchmark datasets and a simulation study based on the scheme by Pledger (2005), see Section 8 . Across the variety of settings we have discussed, the performance of the proposed 
estimators of the upper bound is reasonably successful, as at least one of the two gives a reliable upper bound for the unknown population size. From the results of the simulation experiment, we observe that $\widehat{N}_{\star}^{u}$ is always very close to or larger than the true population size, and in the majority of the analyzed samples it produces a bound that is larger than the population size. When this is not the case we may rely on $\hat{N}_{c}^{u}$, which always closely resembles the true upper bound for the population at hand. There is a further argument as to why the proposed ordinal approach is appropriate in a capture-recapture setting. In fact, conventionally, only the lower counts in the observed truncated distribution are used to get an estimate for the unknown population size. This is motivated by the higher counts being more sensitive to outliers and containing less information. In the proposed ordinal approach, however, we use the cumulative distribution function and, therefore, make use of the higher counts in a more robust framework. An additional reason to consider the proposed approximate upper bound is that it can be used in a Bayesian setting to define a more detailed prior for $N$, see, for example, Farcomeni and Tardella (2010), King et al. (2014), Alunni Fegatelli et al. (2017). One possible route to develop such an approach is based on following the advice given by Garthwaite et al. (2005), who discuss the elicitation of prior distributions based on expert knowledge.

\section{OPEN RESEARCH BADGES}

\section{(II) 2}

This article has earned Open Data and Open Materials badges. Data and materials are available at https://osf.io/y8vtk.

\section{DATA AVAILABILITY STATEMENT}

The data that support the findings in this paper are available in the Supporting Information of this article.

\section{ORCID}

Marco Alfò (D) https://orcid.org/0000-0001-7651-6052

Dankmar Böhning

https://orcid.org/0000-0003-0638-7106

\section{REFEREN CES}

Al Aamri, A.K. (2018) Quantifying the patterns of road traffic crashes in the sultanate of Oman: Statistical evaluation of aggregate data from police records. PhD Thesis, University of Southampton.

Alunni Fegatelli, D., Farcomeni, A. and Tardella, L. (2017) Bayesian population size estimation with censored counts. In: Böhning, D., van der Hejiden, P.G.M. and Bunge, J. (Eds.) Capture-Recapture Methods for the Social and Medical Sciences. New York, NY: Chapman and Hall/CRC. Chapter 25, 371-385.

Böhning, D., Suppawattanabodee, B., Kusolvisitkul, W. and Viwatwongkasem, C. (2004) Estimating the number of drug users in Bangkok 2001: a capture-recapture approach using repeated entries in one list. European Journal of Epidemiology, 19, 1075-1083.
Borchers, D.L., Buckland, S.T. and Zucchini, W. (2004) Estimating Animal Abundance: Closed Populations. London: Springer.

Bunge, J. and Fitzpatrick, M. (1993) Estimating the number of species: a review. Journal of the American Statistical Association, 88, 364-373.

Bunge, J., Woodard, L., Böhning, D., Foster, J.A., Connolly, S. and Allen, H.K. (2012) Estimating population diversity with CatchAll. Bioinformatics, 28, 1045-1047.

Carothers, A.D. (1973) Capture-recapture methods applied to a population with unknown parameters. Journal of Animal Ecology, 42, 125146.

Chao, A. (1984) Nonparametric estimation of the number of classes in a population. Scandinavian Journal of Statistics, 11, 265-270.

Chao, A. (1987) Estimating the population size for capture-recapture data with unequal catchability. Biometrics, 43, 783-791.

Chao, A. (1989) Estimating population size for sparse data in capturerecapture experiments. Biometrics, 45, 427-438.

Chao, A. (2001) An overview of closed capture-recapture models. Journal of Agricultural, Biological, and Environmental Statistics, 6, 158175.

Chao, A., Tsay, P.K., Lin, S.-H., Shau, W.-Y. and Chao, D.-Y. (2001) The applications of capture-recapture models to epidemiological data. Statistics in Medicine, 20, 3123-3157.

Edwards, W.R. and Eberhardt, L.L. (1967) Estimating cottontail abundance from live trapping data. Journal of Wildlife Management, 31, 87-96.

Efron, B. and Thisted, R. (1976) Estimating the number of unseen species: How many words did Shakespeare know? Biometrika, 63, 435-447.

Eren, M.I., Chao, A., Hwang, W.-H. and Colwell, R.K. (2012) Estimating the richness of a population when the maximum number of classes is fixed: a nonparametric solution to an archaeological problem. PLoS ONE, 7, e34179.

Farcomeni, A. and Tardella, L. (2010) Reference Bayesian methods for recapture models with heterogeneity. Test, 19, 187-208.

Garthwaite, P.H., Kadane, J.B. and O'Hagan, A. (2005) Statistical methods for eliciting probability distributions. Journal of the American Statistical Association, 100, 680-701.

van der Heijden, P.G.M., Cruyff, M.J.L.F., van Howelingen, H.C. (2003) Estimating the size of a criminal population from police records using the truncated Poisson model. Statistica Neerlandica, 57, 1-16.

King, R., Bird, S.M., Overstall, A.M., Hay, G. and Hutchinson, S.J. (2014) Estimating prevalence of injecting drug users and associated heroin-related death rates in England by using regional data and incorporating prior information. Journal of the Royal Statistical Society, Series A, 177, 209-236.

Lanumteang, K. and Böhning, D. (2011) An extension of Chao's estimator of population size based on the first three capture frequency counts. Computational Statistics and Data Analysis, 55, 2302-2311.

Link, W.A. (2003) Nonidentifiability of population size from capturerecapture data with heterogeneous detection probabilities. Biometrics, 59, 1123-1130.

Liu, G., Rong, G., Zhang, H. and Shan, Q. (2015) The adoption of capture-recapture in software engineering: a systematic literature review. EASE '15 Proceedings of the 19th International Conference on Evaluation and Assessment in Software Engineering, pp. $1-13$.

Lloyd, C.J. and Frommer, D. (2004a) Estimating the false negative fraction for a multiple screening test for bowel cancer when negatives are 
not verified. Australian and New Zealand Journal of Statistics, 46, 531-542.

Lloyd, C.J. and Frommer, D. (2004b) Regression based estimation of the false negative fraction when multiple negatives are unverified. Journal of the Royal Statistical Society, Series C, 53, 619-631.

Lloyd, C.J. and Frommer, D. (2008) An application of multinomial logistic regression to estimating performance of a multiple-screening test with incomplete verification. Journal of the Royal Statistical Society, Series C, 57, 89-102.

Mao, C.X. (2007) Estimating population sizes for capture-recapture sampling with binomial mixtures. Computational Statistics and Data Analysis, 51, 5211-5219.

Mao, C.X. and Lindsay, B.G. (2007) Estimating the number of classes. Annals of Statistics, 35, 917-930.

Pledger, S.A. (2005) The performance of mixture models in heterogeneous closed population capture-recapture. Biometrics, 61, 868-876.

Rocchetti, I., Bunge, J. and Böhning, D. (2011) Population size estimation based upon ratios of recapture probabilities. Annals of Applied Statistics, 5, 1512-1533.

Rocchetti, I., Alfò, M. and Böhning, D. (2014) A regression estimator for mixed binomial capture-recapture data. Journal of Statistical Planning and Inference, 145, 165-178.

Sanathanan, L. (1977) Estimating the size of a truncated sample. Journal of the American Statistical Association, 72, 669-672.
Software Testing Help. Available at: https://www.softwaretestinghelp. com/tips-to-find-valid-defects-in-any-application/ [Accessed 27 January 2020].

Wilson, R.M. and Collins, M.F. (1992) Capture-recapture estimation with samples of size one using frequency data. Biometrika, 79, 543553.

\section{SUPPORTING INFORMATION}

Web appendices and Tables referenced in Sections 1 and 6 are available with this paper at the Biometrics website on Wiley Online Library. A simple R code and the analyzed data are given to perform the estimation and the standard error calculation for the approximate estimators of the upper bound

\section{How to cite this article: Alfò M, Böhning D,}

Rocchetti I. Upper bound estimators of the population size based on ordinal models for capture-recapture experiments. Biometrics. 2020;1-12. https://doi.org/10.1111/biom.13265 